\title{
The Interaction of Reflection, Regulatory Abilities and Mental States in the Educational Activities of Students
}

\author{
A.O. Prokhorov (a), A.V. Chernov*(b), M.G. Yusupov (c) \\ (a), (b), (c) Kazan (Volga Region) Federal University, Kazan, Russia
}

\begin{abstract}
The article is devoted to the study of reflective regulation of mental states. Students of the Kazan Federal University of various specialties at the age of 18-20 years participated in this study, in total - 93 people. Various methods of diagnosing reflection, regulatory abilities and mental states of a person were used. The state of optimal intensity is typical for students with medium reflection and high level of self-control, as well as for "low-reflective" students with average self-control indicators. At the same time, the least intense states (depression, sadness) are characteristics of "highly reflective" students in combination with a low level of self-control. Intensity of students' states with low and medium level of ability to plan does not change in the continuum from low to high reflection, in contrast to students with high abilities. The specificity of the relationship between metastrategies and the reflective regulation of states is revealed, which consists in the fact that each metastrategy is characterized by a particular influence on the reflective regulation of states.
\end{abstract}

Keywords: mental state, reflection, regulatory ability, learning activity.

(C) 2019 A.O. Prokhorov, A.V. Chernov, M.G. Yusupov

This is an open access article distributed under the terms of the Creative Commons Attribution License (CC BY 4.0), which permits unrestricted use, distribution, and reproduction in any medium, provided the original author and source are credited.

Published by Kazan federal university and peer-reviewed under responsibility of IFTE-2019 (V

International Forum on Teacher Education)

* Corresponding author. Tel.: +79510641296; e-mail address: albertprofit@mail.ru 


\section{Introduction}

According to the concept of reflective regulation of mental states, the regulatory process takes place with the active participation of the main cognitive processes (perception, ideas, mnemonic processes, thinking, etc.), integral processes (goal setting, anticipation, decision making, forecasting, planning, programming, control, self-control) and metacognitive processes (metaperception, meta-memory, meta-thinking, etc.) based on the properties of the personality (temperament, character, etc.) and meta-properties. The incompatibility of experienced states with situations of vital activity "triggers" basic, integral and metacognitive processes, which result in awareness, judgment and rethinking, together with subsequent planning, forecasting, elaboration of strategies, decision making and actualization of operational means of self-regulation, as well as their further testing (metacognitive strategies) (Prokhorov, Chernov, \&Yusupov, 2016).

\section{Purpose and objectives of the study}

To identify the relationship between reflection, regulatory abilities (metastrategies) and mental states, a study was conducted.

\section{Literature review}

International psychology places the concept of "metacognitive awareness" at the center of its research. That is why, the human intellect receives a new quality called "cognitive monitoring" by Flavell (1976). In other words, this is the ability to introspectively analyze and track the course of one's own intellectual activity from its individual aspects (Flavell, 1976). A similar concept of "selfmonitoring", as personality traits, was introduced by Snyder (1974). The concept also includes the ability to register, through self-observation and self-regulation, one's expressive behavior and selfpresentation in new situations, as well as the implementation of this ability, namely, the management of the impression made on people.

Grant (2001) proposed a variant of determining the propensity for self-analysis through the concept of "metacognition". In his opinion, this propensity should not be measured by itself, but through an assessment of the metacognitive processes of reflection. It was found that a high propensity for self-analysis is associated with flexibility, the internal locus of control and realistic thinking.

In Paris and Winograd (1990) conception it is convincingly shown that students can improve their learning by studying their own thinking. Teachers, in this case, provide knowledge, telling students about effective problem-solving strategies, explaining the cognitive and motivational characteristics of thinking. Researchers who have studied the field of metacognition (knowledge of their knowledge) note that learning meta-cognitive processes will improve learning outcomes.

\section{Methodology}

Participants were the students of Kazan Federal University at the age of 18-20 years, 93 people in total.

In the course of the study, the following methods were used: 1 . The technique of the level of expression and directionality of reflection by Grant (2002, as cited in Karpov and Skityaeva, 2005), including scales of social reflection and self-reflection, as well as a general measure of the severity 
of self-reflective awareness; 2 . The technique of self-assessment of metacognitive behavior by Costa (1987, as cited in Karpov and Skityaeva, 2005), consisting of statements that allow revealing the frequency of using 12 different metastrategies of behavior. This technique is best suited for diagnosis in the process of learning activities, because it takes into account various strategies used in the process of cognition. 3. The method "Relief of a mental state" by Prokhorov (2004). 4. Everson and Tobias's (2007) questionnaire, which includes scales: metacognitive involvement in activities, the use of metacognitive strategies, action planning and self-testing (as cited in Karpov and Skityaeva, 2005). 5. The method "Style of behavior self-regulation" (Morosanova, 1998). 6. The method "Anticipative ability" (prognostic competence) by Mendelevich (1996). Multivariate analysis of variance ANOVA, and averages analysis were used in the study.

\section{Findings}

Based on the analysis of variance, a model was constructed for the interaction of mental states and reflection depending on the level of regulatory abilities, where the average for all four substructures of mental states acts as a dependent variable. The selected model of analysis of variance is statistically significant at the level of $p<0.001$, which is extremely high, and explains $34 \%$ of the variance of mean values of mental states.

Thus, this confirms the assumption that the variables we selected have a statistically significant cumulative effect on the average intensity of mental states. Statistically significant (according to Fisher's F-test) are the effects of the general level of regulatory abilities ( $p<0.001)$, as well as the reflection of activity $(p=0.05)$. According to the above ideas, the reflection of activity can be attributed to cognitive reflection, while the general level of regulatory abilities can be attributed to the regulatory component (Karpov and Skityaeva, 2005).

According to the results of the study, the influence of the interaction of the variables "reflection of activity" and "general level of regulatory abilities" was established both on the average characteristics of the states in all four substructures, and on each individual component. The results of the study of the influence of these variables on the substructures of mental states are presented in Table 1. According to it, the cumulative effect of reflection and the level of regulatory abilities on the experiential substructure $(p<0.001)$ and the average value for all substructures $(p<0.002)$ reaches the greatest significance level. The impact on the remaining components of mental states also reaches a high level of significance.

Table 1

\section{The interaction of reflection activities and the level of regulatory abilities on the} substructure of mental states

\begin{tabular}{|c|c|c|c|c|c|c|}
\hline $\begin{array}{r}\text { Source } \\
\text { of variability }\end{array}$ & $\begin{array}{l}\text { Dependent } \\
\text { variable (mental } \\
\text { state structure) }\end{array}$ & $\begin{array}{l}\text { Sum } \\
\text { of squares } \\
d f\end{array}$ & $f$ & $\begin{array}{r}\text { Mean } \\
\text { square }(M S)\end{array}$ & $\begin{array}{l}\text { criteria }^{F-} \\
\text { value }\end{array}$ & $\begin{array}{l}\quad P \\
\text {-level } \\
\text { criterion }\end{array}$ \\
\hline $\begin{array}{l}\text { Genera } \\
\text { 1 Level of }\end{array}$ & $\begin{array}{l}\text { Mean of } \\
\text { substructures }\end{array}$ & $\begin{array}{l}4780 \\
, 388\end{array}$ & & $\begin{array}{l}1195, \\
097\end{array}$ & $78^{4,4}$ & 002 \\
\hline $\begin{array}{l}\text { regulatory } \\
\text { abilities X }\end{array}$ & $\begin{array}{l}\text { Psychologic } \\
\text { al processes }\end{array}$ & $\begin{array}{l}4795 \\
, 023\end{array}$ & & $\begin{array}{l}1198, \\
756\end{array}$ & $29^{3,4}$ & 009 \\
\hline
\end{tabular}




\begin{tabular}{|c|c|c|c|c|c|}
\hline \multirow[t]{3}{*}{$\begin{array}{l}\text { reflection of } \\
\text { activity }\end{array}$} & $\begin{array}{l}\text { Physiologic } \\
\text { al reactions }\end{array}$ & $\begin{array}{l}2825 \\
, 640\end{array}$ & $\begin{array}{l}{ }^{706,4} \\
10^{70}\end{array}$ & $19^{3,2}$ & 013 \\
\hline & Experience & $\begin{array}{r}9211 \\
, 735\end{array}$ & $\begin{array}{l}2302, \\
934\end{array}$ & $19^{4,7}$ & 001 \\
\hline & Behavior & $\begin{array}{l}4229 \\
, 533\end{array}$ & $\begin{array}{l}1057, \\
383\end{array}$ & $55^{3,1}$ & 015 \\
\hline
\end{tabular}

Referring to Figure 1, which reflects the change in the intensity of mental states, depending on the severity of reflection of activity and the level of regulatory abilities. People with a high level of regulatory abilities, regardless of the level of reflection, experience high-intensity states. In the case of an average level of regulatory abilities, the maximum intensity of a state is achieved with average indicators of the expression of reflection. In turn, as the level of reflection increases in combination with low levels of regulatory abilities, the intensity of mental states decreases.

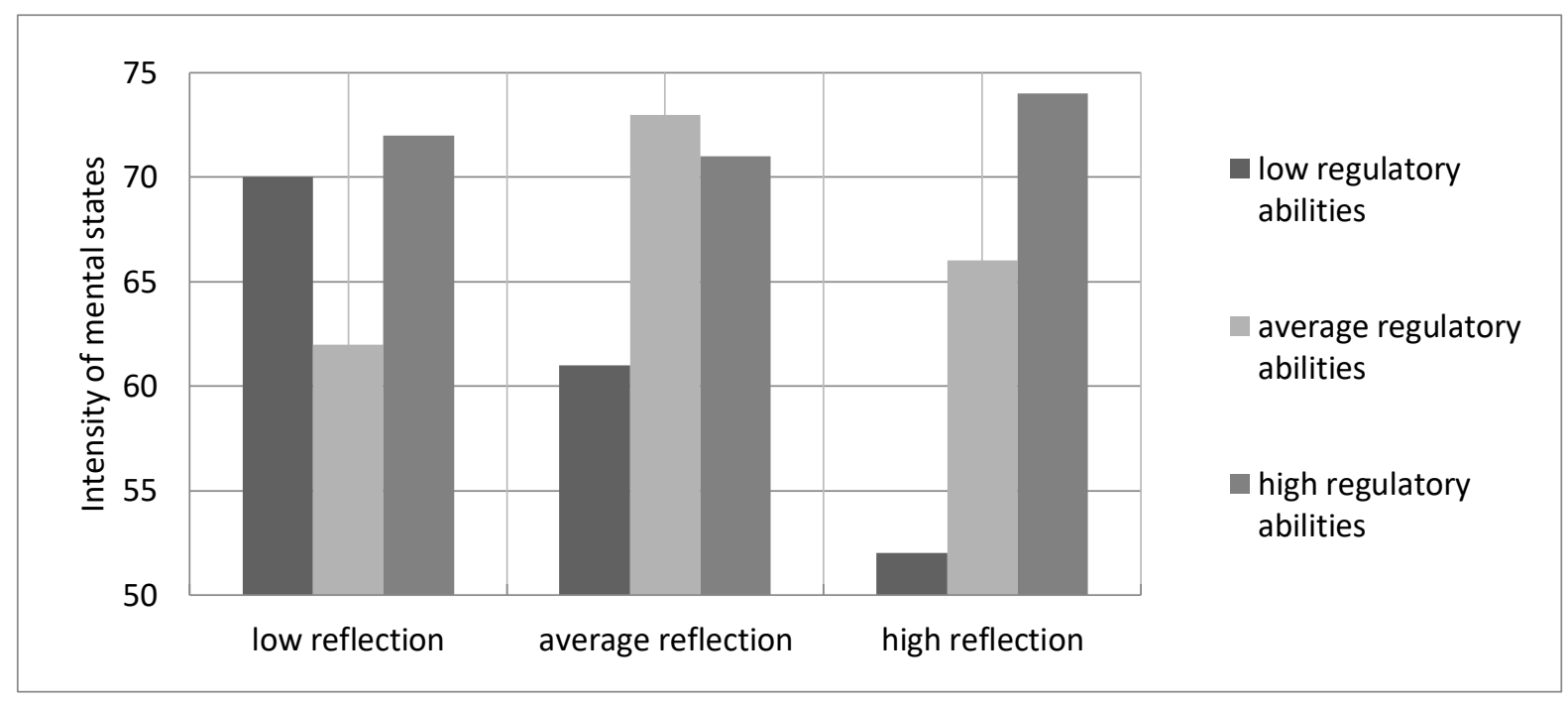

Fig. 1. Influence of the interaction of reflection and level of regulatory abilities on the intensity of mental states

Thus, the most intense mental states are reached by individuals with the same indicators of the level of regulatory abilities and reflection: high regulatory ability with high reflection and low regulatory ability - with low reflection. In general, a decrease in the level of regulatory abilities with high reflection is an important condition for reducing the intensity of the conditions experienced. Consequently, a key indicator affecting mental states is the level of the subject's regulatory abilities, and reflection, in turn, has a transformational function, being a kind of "moderator" (enhancing or weakening) the interaction of other variables.

In general, we note that when analyzing the substructures of mental states as a dependent variable, it was found that the analysis of variance is statistically significant at the level of $p \leq 0.001$ and explains $40 \%$ of the variance of their average characteristics. According to the Fisher's $\mathrm{F}$ criterion, only the variable reflection of activity statistically significant affects the intensity of students' mental states in a tense situation $(\mathrm{p} \leq 0.002)$. But the interaction of the indicators of "reflection of activity" and "general level of self-control" ( $\mathrm{p} \leq 0.003)$ also significantly influences changes in mental states. 
Comparing the characteristics of the influence of these factors on students' mental states, we designate that optimal (high) intensity states are typical for students with medium reflection and high levels of self-control, as well as for "low-reflexive" students with average self-control indicators. At the same time, the least intense states (depression, sadness) are characteristics of "highly reflexive" students in combination with a low level of self-control.

As a result of surveys, it was found that the intensity of conditions in people with low and medium levels of planning ability remains unchanged in the continuum from low to high reflection, while the intensity of conditions in people with high planning abilities increases with growth of the reflectivity level. High-intensity states are inherent in low-reflective subjects with average scores of results. However, as reflection rises, individuals with an average ability to evaluate results experience less intense states.

As a result of data processing using analysis of variance, it was found that the effect of anticipation is statistically significant at the level of $p \leq 0.008$ and explains $67 \%$ of the variance of average values of mental states. The effects of self-reflection indicators (awareness of one's own experiences) and the personal property of predictability $(\mathrm{p} \leq 0.004)$ are also statistically significant (according to Fisher's F-criterion). People with low predictive ability, regardless of the reflectivity index, demonstrate the highest intensity of all indicators of mental states. Students with a low level of self-reflection and high characteristics of anticipation also experience states of high intensity, which is not observed in individuals with high reflection of their experiences: their condition is characterized by low values.

The analysis of the relationship between the awareness of one's own experiences, mental states and chrono-rhythmology anticipation, which serves as an integral process, shows that the interaction of indicators of self-reflection and temporary anticipation is significant at the level of $\mathrm{p} \leq 0.004$. People, who are well oriented in time, demonstrate a state of high intensity only in the case of a low level of reflection. At the same time, the conversion of consciousness to one's mental state, an attempt to realize it, leads to a decrease in the indicators of mental state in all substructures. In the case of low temporal prognosis, on the contrary, there is a slight increase in the intensity of states as the level of reflection increases.

As a result of the analysis, it was established that the following metastrategies of human behavior (according to Costa, 1987, as cited in Karpov and Skityaeva, 2005), influence the reflective state regulation during training activities: conscious decision making ( $p \leq 0.020$ - according to Fisher's criterion), where the relationship is established as with separate state substructures (experiences, physiological reactions, behavior), and with the mean value of all substructures in general; understanding the results obtained $(\mathrm{p} \leq 0.050)$; determining the exact wording $(\mathrm{p} \leq 0.043)$; playing the position of the interlocutor $(\mathrm{p} \leq 0.049)$, a written fixation of one's own thoughts $(\mathrm{p} \leq 0.050)$, as well as the construction of mental representations of experience $(\mathrm{p} \leq 0.007)$.

The study found that in the case of a low level of degree of the strategy of conscious decision making, mental activity is expressed rather weakly, and as awareness of one's own experiences grows, it only decreases further. With a high degree of this strategy, a significant increase in the intensity of the state is observed with an increase in the level of reflection of the subject. It is with high reflection that this strategy turns out to be the most productive. 
Although, it is worth noting that with low reflection rates, the intensity of the states is completely identical for different groups of people with different metastrategies. We also note that a similar pattern is a characteristic of all substructures of students' states in a class.

When using the strategy of active understanding of the results obtained, a high activity of the state is observed, and it grows with an increase in the reflection index (from 72 to 79 points according to the "Relief of a mental state" technique). In turn, the low inclusiveness of this strategy in the regulatory process with low reflection leads to extremely high states (an average of 80 points). The inverse tendency was found with high reflection: here, immersion in one's experiences reduces the indicators of states to its minimum (65 points).

The metastrategy of formulating precise definitions and explaining vague notions operates in completely different ways, depending on the level of reflection of one's own experiences by the subject. If at low reflection, the intensity of states turns out to be somewhat higher with the active use of this strategy of behavior, then at high rates it is quite opposite - the less often a person uses this strategy, the more optimal states he experiences. Here the following tendency is found: the high reflectivity of one's experiences with the frequent use of metastrategies reduces the intensity of the state, while the low one becomes the condition for its growth.

Frequent use of the strategy of playing the position of the interlocutor, a mental dialogue with him, in its own way influences the reflexive regulation of mental states. In the case of frequent use of this strategy, the intensity of the states slightly decreases in the range from low to high reflection, that is, the severity of the state of highly reflective subjects is lower than that of low reflective ones. However, a significant "jump" in the intensity of the experienced states is characteristic of those who rarely use this strategy of behavior in the process of learning activities: in the range from low reflection to high intensity, the experience increases from 66 to 84 points. The rare use of lowreflective strategy especially affects the "behavior" substructure, it becomes less open, adequate and thoughtful.

We consider the strategy of writing own thoughts and ideas in the regulation of states by people with different levels of reflection. Opposite results were found here: with the active use of this strategy, the activity of mental states decreases significantly with an increase in the level of reflection and, conversely, ignoring this metastrategy leads to an increase in the intensity of states. This strategy affects, first of all, the substructure of the behavior.

One of the most frequently used metastrategies and, at the same time, influencing the reflective regulation of states was the strategy of building mental experience: a relationship was found with all the substructures of the selected states (it is especially significant when it influences the indicators of experience and behavior). With the active use of this metastrategy, the intensity of the state remains high regardless of the indicator of the reflection level. However, in the case of a rare use of the strategy, the intensity of the experienced states increases significantly with an increase in the reflection of the experiences (from 40 to 82 points), that is, the state of a low level of activity changes to a state of high intensity and reaches the same level as when using the strategy. Thus, here reflection serves as a facilitator of a first-order dependency. The active use of the strategy of building mental experience and a high level of reflection are a necessary condition for the regulation of mental states. 
Using the Everson and Tobias's (2007, as cited in Karpov and Skityaeva, 2005) we were able to detect the effect of action planning and self-test strategies $(p \leq 0.050)$ on the reflective regulation of mental states. Here, highly reflective subjects are characterized by a high intensity of state with a rare use of an action planning strategy and the lowest intensity possible - with its frequent use. A mirror opposite is observed for low-reflexive individuals: their states are most intense when using this strategy.

The situation with the self-testing strategy is somewhat different: with its rare application, the intensity of the states remains unchanged, regardless of the level of reflection. If the strategy is used frequently, then with increasing reflection, the level of activity of the mental state also increases (from 69 to 82 points).

\section{Discussions}

Lyke (2009) put forward and tested the hypothesis that (1) people with higher levels of awareness will be happier and more satisfied with their lives than people with lower levels and (2) that the interaction between self-reflection and awareness affects both happiness, and life satisfaction. Brown and Brown (2011) found that the more people think about their attitude to a problem, the more they experience the negative states.

Researchers believed that taking into account the specifics of reflection would improve the learning process. They identified several types of reflection that increase the level of creative thinking of students. The authors found that if a student himself can solve the problem, then for a successful mental process, only the inclusion of intellectual reflection is needed (Semenov and Stepanov, 2008).

\section{Conclusion}

As a result of surveys, it was found that the intensity of conditions in people with low and medium levels of planning ability remains unchanged in the continuum from low to high reflection, while the intensity of conditions in people with high planning abilities increases with the level of reflectivity. High-intensity states are inherent in low-reflective subjects with average scores of results. However, as reflection rises, individuals with an average ability to evaluate results experience less intense states.

Summing up the study of integral and metacognitive processes in the reflexive regulation of mental states, we note that the reflexive regulation of states is connected and mediated by these processes. The latter is specific in each case of influence. In particular, the intensity of the states of people with low and medium levels of ability to plan does not change in the continuum from low to high reflection, in contrast to persons with high abilities.

The specificity of the relationship between metastrategies and the reflexive regulation of states is revealed, which consists in the fact that each metastrategy is characterized by a peculiar influence on the reflective regulation of states. In some cases, the influence of metastrategies is more prominent (decision-making, strategy for building mental experience, etc.), in others - less prominent (formulating precise definitions, playing the position of the interlocutor, etc.). 


\section{Acknowledgments}

The research was conducted with the support of the Russian Foundation for Basic Researches, project No 17-06-00057a.

This work was funded by the subsidy allocated to Kazan Federal University for the state assignment in the sphere of scientific activities.

\section{References}

Brown, J., \& Brown, M. (2011). Self-reflection and feelings of self-worth: When Rosenberg meets Heisenberg. Journal of Experimental Social Psychology, 47, 1269-1275.

Brown, K. W., \& Ryan, R. M. (2003). The benefits of being present: Mindfulness and its role in psychological well-being. Journal of Personality and Social Psychology, 84(4), 822-848.

Flavell, J. H. (1976). Metacognitive aspects of problem solving. In L. B. Resnick (Ed.), The nature of intelligence (pp. 231-235). Hillsdale, NJ: Lawrence Erlbaum.

Grant, A. M. (2001). Rethinking psychological mindedness: metacognition, self-reflection, and insight. Behaviour Change, 18, 8-17.

Karpov, A. V., \& Skityaeva, I. M. (2005). Psychology of metacognitive processes of personality. Moscow: Institute of Psychology of the Russian Academy of Sciences.

Lyke, J. (2009). Insight, but not self-reflection, is related to subjective well-being. Personality and Individual Differences, 46, 66-70.

Mendelevich, V. D. (1996). Antiction mechanisms of neurosis genesis. Psychological journal, 4(17), $107-115$.

Morosanova, V. I. (1995). Individual style of self-regulation in the arbitrary activity of a person. Psyhological Journal, 4, 26-35.

Paris, S. G., \& Winograd, P. W. (1990). How metacognition can promote academic learning and instruction. In B.J. Jones, \& L. Idol (Eds.), Dimensions of thinking and cognitive instruction (pp. 15-51). Hillsdale, NJ: Lawrence Erlbaum Associates.

Prokhorov, A. O., Chernov, A. V., \& Yusupov, M. G. (2016). On the mechanism of interaction between mental states and cognitive processes in academic activity of students. Mathematics Education, 11(4), 569-581.

Prokhorov, A. O. (2004). Methods of diagnosis and measurement of mental states of the person. Moscow: Per SE.

Prokhorov, A. O., \& Chernov, A. V. (2016). Reflection and mental state in different forms of learning activity of students. Psychological Journal, 37(6), 47-56.

Schraw, G., \& Dennison, R. S. (1994). Assessing Metacognitive Awareness. Contemporary Educational Psychology, 19, 460-475.

Snyder, M. (1974). Self-monitoring of expressive behavior. Journal of Personality and Social Psychology, 30, 526-537.

Stepanov, S. Yu., \& Semenov, I.N. (1985). The psychology of reflection: problems and research. Questions of Psychology, 3, 31-40. 